\title{
Designing a Reverse Logistics Network for End-of-Life Vehicles Recovery
}

\author{
Masoud Zarei, Saeed Mansour, Ali Husseinzadeh Kashan, \\ and Behrooz Karimi
}

Department of Industrial Engineering, Amirkabir University of Technology, 424 Hafez Avenue, 1591634311 Tehran, Iran

Correspondence should be addressed to Saeed Mansour, s.mansour@aut.ac.ir

Received 28 March 2009; Revised 30 September 2009; Accepted 12 January 2010

Academic Editor: Nickolas S. Sapidis

Copyright @ 2010 Masoud Zarei et al. This is an open access article distributed under the Creative Commons Attribution License, which permits unrestricted use, distribution, and reproduction in any medium, provided the original work is properly cited.

The environmental factors are receiving increasing attention in different life cycle stages of products. When a product reaches its End-Of-Life (EOL) stage, the management of its recovery process is affected by the environmental and also economical factors. Selecting efficient methods for the collection and recovery of EOL products has become an important issue. The European Union Directive 2000/53/EC extends the responsibility of the vehicle manufacturers to the postconsumer stage of the vehicle. In order to fulfill the requirements of this Directive and also efficient management of the whole recovery process, the conceptual framework of a reverse logistics network is presented. The distribution of new vehicles in an area and also collecting the End-of-Life Vehicles (ELVs) and their recovery are considered jointly. It is assumed that the new vehicles distributors are also responsible for collecting the ELVs. Then a mathematical model is developed which minimizes the costs of setting up the network and also the relevant transportation costs. Because of the complexity of the model, a solution methodology based on the genetic algorithm is designed which enables achieving good quality solutions in a reasonable algorithm run time.

\section{Introduction}

Economical, social, and environmental dimensions are three main pillars of sustainable development. The environmental factors have been mainly taken into account in the developed countries, while in the developing countries the economical issues have been the main concern. However issues such as air and water pollution and increased public concern on these problems, resources scarcity, and international legislations have increased the importance of environmental topic in the developing countries.

The Environmental Conscious Manufacturing (ECM) and its related Design For Environment (DFE) guidelines are getting more and more attention. ECM involves 
producing products such that their overall negative environmental effects are minimized [1]. For doing this, the whole life cycle of the products should be considered.

When a product reaches its End-Of-Life stage, it would usually have potential for polluting the environment. On the other hand, EOL products may have valuable parts, components, and materials which could be used again or returned to the production cycle. That is because the EOL products recovery is an important stage in products life cycle.

Vehicle is a complex product composed of thousands parts with different types of materials; so the ELV recovery is a complicated process. Based on the quality of the ELV's parts and also the economical considerations, different recovery and disposal options could be selected. These options include reuse, remanufacture, material recycling, incineration, and landfill. The last two options have more negative environmental effects.

As ELVs recovery is a very important issue, therefore the European Union has set up the Directive 2000/53/EC [2] for this particular problem. Based on this Directive, the responsibility of the manufacturer extends to the post-consumer stage of the vehicle. The Directive also sets some recovery and recycling targets. Minimizing the costs of collecting the ELVs and delivering them to suitable treatment facilities leads to a reverse logistics network design problem. Reverse logistics is defined as "the process of planning, implementing, and controlling the efficient, cost effective flow of raw materials, in-process inventory, finished goods and related information from the point of consumption to the point of origin for the purpose of recapturing value or proper disposal" [3].

The vehicle manufacturers may need to set up an efficient recovery network in order to fulfill the Directive 2000/53/EC requirements. They may also outsource the take back and recovery activities and its management. For instance, in the UK, the producers have contract with the network of administrators. On behalf of the manufacturers they are responsible for the free take-back (from the beginning of 2007), depollution, dismantling, and forwarding the ELV's hulk to the treatment facilities for shredding [4].

The importance of management of the ELVs in the developing countries where the number of vehicles on the roads is increasing at an alarming rate is also becoming more apparent. The implementation of strict product-oriented legislation will sooner or later become of paramount interest in developing countries [5].

In this paper, a reverse logistics network for the management of the ELVs recovery process is designed. Based on a conceptual model, a mathematical model for minimizing the costs of collecting the ELVs and flow of materials between treatment facilities is developed. The distribution of new vehicles and collecting the ELVs are considered simultaneously in the model and performed in the same facilities. Advantages of building such integrated facilities might include cost savings and pollution reduction as results of sharing material handling equipment and infrastructure [6]. A genetic algorithm has also been adapted for solving the model and getting a good solution in a reasonable run time.

\section{Literature Survey}

Research areas related to End-of-Life products and ELVs are extremely diverse. Some researches have concentrated on the Optimal Recovery Plan and Disassembly Sequence for EOL products. In such problems the objective is to determine to what extent the return products must be disassembled and which recovery and disposal options should be applied [7].

Technical-cost models of ELV processing operators were developed and the results obtained show that the recycling targets defined in the EU Directive for 2006 require the removal of an increased number of plastic parts from an ELV [8]. In particular, removing up 
to $14 \%$ of the ELV mass results in a recycling rate over $80 \%$, even for vehicles with a ferrous content up to about $65 \%$. A simulation model for examining the effect of future changes in vehicle material composition on the automotive recycling infrastructure has been developed [9]. The results showed that even in the case of significantly higher rates of dismantling and plastics recovery, the recycling target of $95 \%$ by 2015 seems unattainable without dramatic and fundamental changes, especially in vehicle material composition.

A demanufacturing optimization model has been applied to the ELV recovery process problem where different scenarios including current ELV recycling in the North America and implementing the EU Directive are discussed [10]. The optimal recovery strategies for different kinds of ELVs have also been derived. In another research, innovation in recycling, increased hazardous substance removal, and improved information dissemination have been stated as the results of implementing the ELV Directive [11]. Increasing the level of reuse and remanufacturing has also been proposed as a key part of moving toward sustainable vehicle production.

An expanding research area related to EoL products is designing reverse logistics networks and developing associated mathematical models. The traditional supply chain models have been extended to incorporate environmental factors and used products flow from customers to manufacturers or recyclers. Some researches have concentrated only on used products reverse flow while some other works have considered new products and used products flows simultaneously.

Strategic network design for the ELVs collection problem in Mexico has been studied [5]. In this paper, collection facilities are assumed to perform take-back, depollution, and dismantling activities, and their locations are determined by the model. Three scenarios regarding $100 \%, 90 \%$, and $75 \%$ of coverage for ELV collection demand are also considered. The analysis identified transport costs as a determinant factor for the collection network design. An integrated logistics network is considered for EOL computer products recovery in which a type of hybrid processing facility is assumed [6]. Both forward products and EOL returned products are transferred via these facilities. The objective of the model is to select the locations of the hybrid processing facilities and determine the quantities of the forward and EOL returned products shipped among the network.

A facility location model for the reverse logistics systems, in which both direct and reverse flows have to be considered simultaneously, is presented [12]. The model has been developed for the case of a remanufacturing system. The results showed that reverse flows influence the decisions about facilities locations and flow allocations. This influence varies with the magnitude of the reverse flows, their distribution at demand sites, and also their correlation with forward flows. A mixed integer linear programming model for recycling of sand with the objective of locating regional depots and treatment facilities and determining the amount of flow of materials between these elements has been developed. Also procedures for finding a lower and upper bound for the solution are presented [13]. A single period reverse logistics model which considers transportation of the used products from some origination sites to collection centers and then to refurbishing sites has been developed. In this work a heuristic solution methodology has also been proposed [14].

A conceptual model for simultaneous location-allocation of facilities for a cost effective and efficient reverse logistics network is developed [15]. Two distinct rework sites including repair and refurbishing centers and remanufacturing centers are considered. An integrated holistic conceptual framework and also detailed solutions for network configuration and design at the topological level are provided. A reverse logistics problem is examined where containers in which products are delivered from a central processing point to customers 
(stops) in one period are available for return to the central point in the following period [16]. Determining how much of the returning materials should be picked up at each stop along a delivery route has been focused in the research. A coordinated reverse logistics management system for the treatment of multisource hazardous wastes in a specific high-technology manufacturing zone is presented [17]. A multiobjective optimization model is formulated to seek optimal solutions with the goal of minimizing both the total operational costs and the corresponding risk-induced penalties of the reverse logistics system. A reverse logistics model for minimizing the cost of a multitime-step, multitype hazardous waste recovery system has been developed and a case study is presented [18].

A facility location-allocation model for the carpet materials has been developed [19]. They have considered regional preprocessing centers (RPCs) for identifying, sorting, separating, and compressing the carpets waste. The main characteristics of the proposed model are that the locations of the RPCs are not determined among preset potential locations but they are calculated on a continuous basis by the model. Two cases in Europe and the US have been studied. A model for locating a new plant by considering both primary and secondary products has been developed where primary product is used for meeting the customers' demands and the secondary product is sent back to the plant site by the user [20]. A case study conducted in Germany has resulted in a reverse logistics model for the ELVs recovery [21]. The model is formulated as a Vehicle Routing Problem, where the objective is to minimize the total transportation costs of the network.

A model for reverse supply chain which considers the collection, recycling, and processing of electronic waste has been developed [22]. The model focuses on a multiple tiers of decision-makers concept. These decision-makers may compete within a tier but at the same time cooperate between the tiers. A general linear mixed integer model for maximizing the profits of a supply chain network by considering development and recycling phases has been derived, which falls within the scope of strategic planning [23].

A stochastic programming approach has been implemented for sand recycling treatment facility location problem [24]. A system dynamic methodology has been used to analyze the effect of the environmental parameters on designing product recovery networks [25], where the impact of different decisions on collecting and recovery of used products can be evaluated through dynamic simulation. For a comprehensive survey on reverse logistics systems' characterization and classification models, the reader is referred to [26, 27].

Due to inherent high complexity of the reverse logistics problems, especially when the forward flows are considered simultaneously, different solution methodologies based on the search algorithms, such as genetic algorithm (GA), have been used in solving these problems. A mathematical model of a remanufacturing system by considering a three-stage logistics network has been presented [28]. A hybrid genetic algorithm is proposed in which priority-based encoding method combined with a new crossover operator has been used for the first two stages. A heuristic approach is also applied in the 3rd stage. After performing numerical experiments and comparing the results with the recent researches, the advantages of the proposed algorithm are presented. One of them is that its implementation is very easy; another one is that after genetic operators, always feasible solutions are obtained.

A nonlinear mixed integer programming model and a genetic algorithm that can solve the reverse logistics problem involving both initial collection points and centralized return centers were presented [29]. In these centralized centers, returned products from retailers or end-customers are collected, sorted, and consolidated into a large shipment destined for manufacturers' or distributors' repair facilities. Computational experimentation revealed that GA presented a promise in solving practical-size problems with 30 customers, 10 potential 


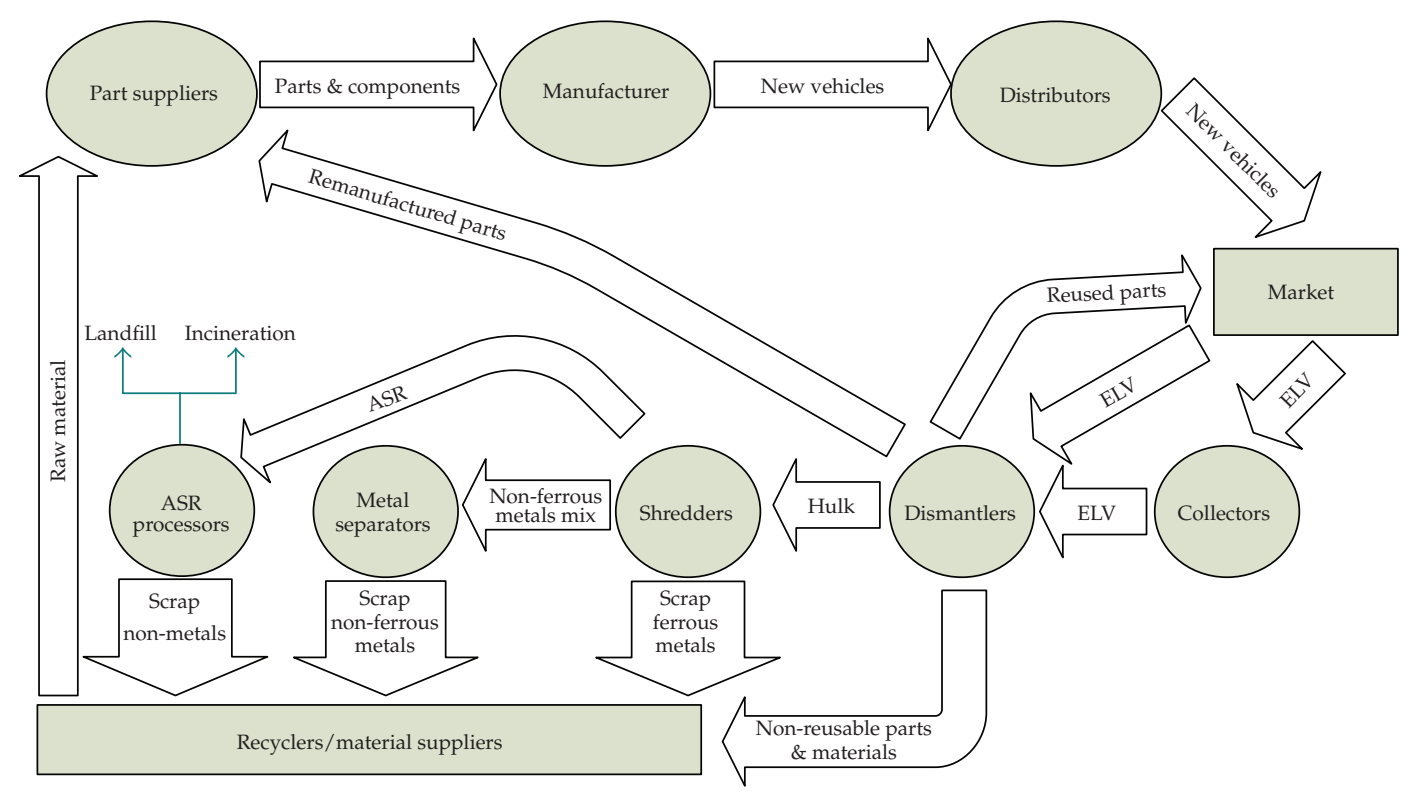

Figure 1: The "Manufacturer Network" Building Blocks.

sites of initial collection points, and five potential sites of centralized return centers. In another work by the same authors, the problem of determining the number and location of initial collection points in a multiple time horizon, while determining the desirable holding time for consolidation of returned products into a large shipment, has been considered [30]. The designed genetic algorithm was tested by an example dealing with products returned from online and retail sales.

A mixed integer nonlinear programming model for the design of a dynamic integrated distribution network to account for the integrated aspect of optimizing the forward and return network simultaneously is given [31]. Then a GA-based heuristic that consists of genetic operations and simplex transshipment algorithm is designed for solving the model. Comparing the solutions of the proposed methodology with the optimal solutions of 18 test problems indicated that the range of gaps with respect to solution quality was from $0.78 \%$ to $8.18 \%$. A genetic algorithm to determine locations of collection points in a reverse logistics network with the purpose of maximizing the coverage of customers is proposed [32]. Also, a mechanism is suggested to count the quantities of collected items in collection points and send the signal to the central return center which leads to increase efficiency of logistics operations. Simulation results indicated that the proposed genetic algorithm is able to produce good-quality solutions. In finding good solutions, it is found that the total number of generations can be set as low as 30 for minimizing the running time.

A facility-location model in a reverse logistics context has been combined with queueing relationships, which enables to account for some dynamic aspects like lead time and inventory positions [33]. Also, the higher degree of uncertainty in reverse logistics problems could be taken into account. Several examples were solved with a genetic algorithm based on the technique of differential evolution. The algorithm found robust solutions within an acceptable time limit that were very close to the true global optimum. The problem of locating collection areas in municipal waste management context has been considered [34]. Two approaches for solving the proposed model based on the genetic algorithm and a heuristic 
have been examined. The quality of the algorithms and their running times are tested in a computational experience with real instances from the metropolitan area of Barcelona.

In this paper, the problem of designing a reverse logistics network for ELVs recovery has been addressed; furthermore, the forward distribution of new cars is also considered. The distribution-collection center entity is then introduced, which its benefit is discussed in Section 4. The available budget in setting up the required facilities is also considered. Due to high complexity of the model, a genetic algorithm methodology is proposed in order to get good solutions in reasonable running time.

\section{Conceptual Model}

In [35], the authors introduced the concept of the "Manufacturer Network". This network includes elements concerning the production of new vehicles and recovery of used ones. Based on the Extended Producer Responsibility (EPR), the manufacturer is responsible for free take back and recovery of its ELVs and must bear all or a significant part of the collection and treatment costs. So the manufacturer would have the central role in this network and needs to supervise the whole treatment process and develop efficient recovery techniques in order to minimize the costs. The building blocks of such network are shown in Figure 1.

\section{Mathematical Model}

In [35], the total network was simplified and separated into two subnetworks, namely, new vehicle production and distribution segment and the ELVs recovery segment. Then based on number of assumptions, the optimization of the ELVs recovery section was done independently.

In this paper it is assumed that the new vehicle distributors are also responsible for collecting the ELVs. These new entities are called distribution-collection centers. The main purpose for considering this assumption is reducing the total cost of the system setup, since there will be no need to establish extra facilities for collecting the ELVs. Based on this assumption, the whole problem network should be optimized as an integrated system and the transportation of the new vehicles and the ELVs will be considered collectively in the model.

\subsection{Assumptions}

The following assumptions have been considered in developing the mathematical model.

(i) The whole problem area is divided into I sections, called demand points. For each of these points, the demand for new vehicle and also the number of ELVs are estimated. All the estimated demands for new vehicles should be materialized and also all the estimated ELVs should be collected and recovered by the network. The developed model could be applied to a geographical area where a manufacturer wants to establish its distribution centers and also collect and recover all its manufactured vehicles that have reached to their end-of-life stage.

(ii) The potential locations for setting up the distribution-collection centers are assumed to be $J$, where the optimal locations of these facilities will be found among these locations by running the proposed model. A budget ceiling for establishing these centers has been considered as a constraint in the developed model. $K$ potential locations are also assumed for setting up the dismantling sites. 
Table 1: Optimal recovery policy for year 2006.

\begin{tabular}{lcc}
\hline Material stream & The ELVs with resale parts & The ELVs without resale parts \\
\hline Fluids & $1.8 \%$ & $1.8 \%$ \\
Tyres & - & $2.5 \%$ \\
Nonresalable plastics parts & $0.3 \%$ & $2 \%$ \\
Reused and remanufactured parts & $59.3 \%$ & - \\
Hulk & $38.6 \%$ & $93.7 \%$ \\
\hline
\end{tabular}

(iii) For each distribution-collection center, the capacities for stocking new vehicles and ELVs may be different and also the costs of setting up these capacities may vary.

(iv) In order to set up each facility, a fixed cost and a variable cost, which is related to its capacity, have been assumed.

(v) It is assumed that reused and remanufactured parts are sold at the dismantling sites. Note that the remanufacturing process is not performed by dismantlers and the disassembled parts and components are sold without any refurbishment. Hence the transportation of the remanufactured parts to the Part Suppliers is not considered. Based on this assumption, the Part Suppliers are not taken into account in the developed model.

(vi) After dismantling, the removed fluids, tyres, and nonresalable plastics are sent to the recyclers. Reused/remanufactured parts and resalable plastic parts are sold at the dismantlers' sites, while the hulk is sent to the shredder. Note that removed hazardous materials are neglected because of their low weight, which is less than 0.1 percent of the vehicle total weight [10].

(vii) The optimal percentages of the above mentioned streams, based on year 2006 requirements (i.e., $85 \%$ of ELV weight recovery with maximum of $5 \%$ energy reclamation) are given in Table 1 [10].

The simplified designed reverse logistics network which is the base of the developed mathematical model is shown in Figure 2.

\subsection{Objective Function}

The model decision variables and parameters have been defined in the appendix. The objective function is given in (4.1):

$$
\begin{gathered}
\operatorname{Min} \sum_{j} H X_{j}+f_{1} \sum_{j} t_{j}+f_{2} \sum_{j} u_{j}+\sum_{k}\left(R Y_{k}+g v_{k}\right)+\sum_{j} t_{j} d_{j} C_{1} \\
+\sum_{i} \sum_{j} t_{1 i j} d_{1 i j} C_{2}+\sum_{i} \sum_{k} t_{2 i k} d_{2 i k} C_{2}+\sum_{j} \sum_{k} t_{3 j k} d_{3 j k} C_{2} \\
+\sum_{n} \sum_{k} \sum_{l} C_{2} d_{4 k l} \theta_{n 5} \beta_{n} t_{4 k l}+\sum_{n} \sum_{k} \sum_{s} C_{2} d_{5 k s} \theta_{n s} \beta_{n} v_{k} .
\end{gathered}
$$




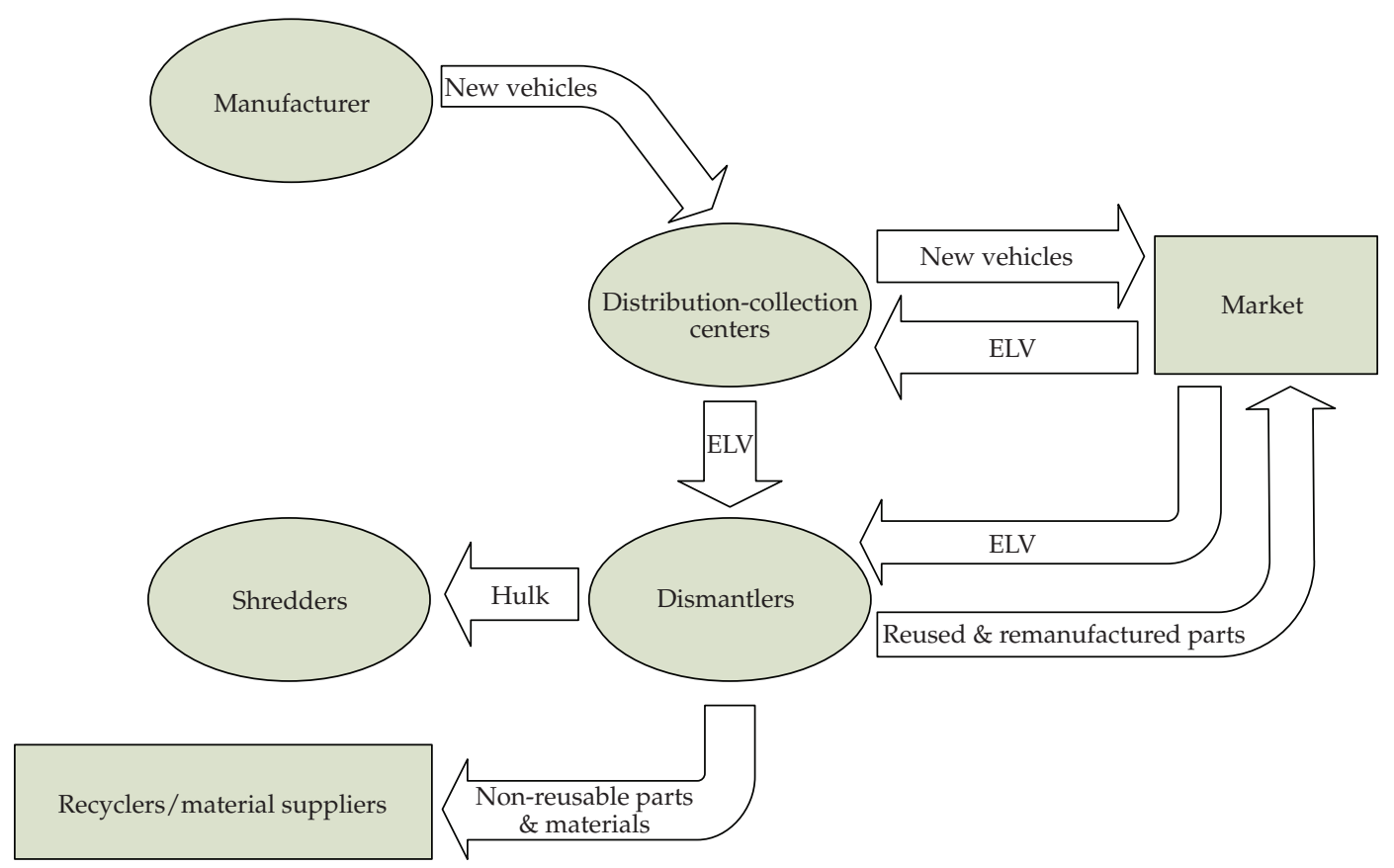

Figure 2: The Simplified Network.

The first term in (4.1) shows the fixed cost of opening the distribution-collection centers. The second and third terms represent the variable costs of opening the distributioncollection centers related to the new vehicles and ELVs, respectively. The opening costs of the dismantling sites will be calculated through the fourth term.

The fifth term in (4.1) represents the transportation cost of the new vehicles from the manufacturer to the distribution-collection centers. The transportation costs of ELVs from the demand points to the distribution-collection centers, from the demand points to the dismantlers, and from the distribution-collection centers to the dismantlers are shown in terms sixth through eighth, respectively.

The last two terms in (4.1) show the flow of materials after dismantling, which consist of transportation of hulks to the shredders and transportation of different material streams to the recyclers.

\subsection{Constraints}

In order to build the mathematical model, the following constraints have been considered.

(i) All the ELVs should be collected from demand points:

$$
\sum_{j} t_{1 i j}+\sum_{k} t_{2 i k}=A_{i} ; \quad \forall i
$$

(ii) Any purchase demands for the new vehicles should be satisfied. The customers may buy their vehicles from any of the distribution-collection centers:

$$
\sum_{j} t_{j} \geq \sum_{i} D_{i}
$$


(iii) The cost of setting up each distribution-collection center should not exceed its determined available budget:

$$
H X_{j}+f_{1} t_{j}+f_{2} u_{j} \leq B_{j} ; \quad \forall j
$$

(iv) ELV capacity of each distribution-collection center is calculated as follows (used for model simplification):

$$
u_{j}=\sum_{i} t_{1 i j} ; \quad \forall j
$$

(v) Dismantler's capacity is calculated as follows (used for model simplification):

$$
v_{k}=\sum_{i} t_{2 i k}+\sum_{j} t_{3 j k} ; \quad \forall k
$$

(vi) Distribution-collection centers' capacity constraints. two maximum capacities related to the new vehicles and ELVs are considered for each of the distributioncollection centers. These capacities are dependent on each location available resources:

$$
\begin{aligned}
& t_{j} \leq C_{j 1} X_{j} ; \quad \forall j, \\
& u_{j} \leq C_{j 2} X_{j} ; \quad \forall j .
\end{aligned}
$$

(vii) Dismantlers' capacity constraints: a maximum capacity for setting up each dismantling site is considered:

$$
v_{k} \leq E_{k} Y_{k} ; \quad \forall k
$$

(viii) Materials flow constraints: transportation of all collected ELVs from distributioncollection centers to dismantlers and transportation of hulks from dismantlers to the shredders:

$$
\begin{aligned}
& u_{j}=\sum_{k} t_{3 j k} ; \quad \forall j, \\
& v_{k}=\sum_{l} t_{4 k l} ; \quad \forall k .
\end{aligned}
$$

(ix) Decision variables sign restrictions:

$$
X_{j}, Y_{k}=0 \text { OR } 1,
$$

All Other Variables Are $\geq 0$. 


\section{Proposed Solution Methodology and Implementation Issues}

With the knowledge that the proposed mathematical model is a general variant of the location-allocation model and hence an NP-hard problem [14], the reverse logistics network design problem does not permit for developing polynomial time exact algorithms. So, it was decided to achieve high-quality solution(s) through using search-based algorithms. Especially the proposed solution methodology was based on the use of genetic algorithm.

Genetic algorithms (GAs) are powerful and broadly applicable stochastic search and optimization techniques based on principles of evolution theory. In the past few years, genetic algorithms have received considerable attention for solving difficult combinatorial optimization problems [36]. GAs start with an initial set of (random) solutions called population. Each individual in the population is called a chromosome, representing a solution to the problem at hand. The chromosomes evolve through successive iterations, called generations. During each generation, the chromosomes are evaluated, using some measures of fitness. Generally speaking, the genetic algorithm is applied to spaces that are too large to be exhaustively searched. In the following the operators that the implementation of GA utilizes to search for high-quality solutions is described.

\subsection{Representation and Fitness Function}

The proper representation of a solution plays a key role in the development of a genetic algorithm. In the proposed coding scheme, each gene corresponds to one of available locations. A solution is partially represented by an array of size $J+K$ as a chromosome. Each gene takes a binary value where 1 denotes the corresponding location considered for the construction of required facility and 0 addresses otherwise.

The adopted representation helps to decide where the required facilities should be located. The intractability of this reverse logistics network design problem comes directly from the discrete nature of the locations related variable. Once the locations are fixed, the remaining problem of deciding the quantity variables becomes tractable since this squeezed problem is simply a continuous linear program. When this linear program is solved optimally the objective value of the corresponding solution is determined. It is considered that this value corresponds to the fitness of the relevant chromosome.

The generated solutions should satisfy all the model constraints. To ensure the feasibility of the generated solutions, it is necessary to verify if the solutions can satisfy constraints (4.2) and (4.3). To do this, every time that a constraint becomes infeasible a new facility is established at the potential sites randomly. This process is continued until getting the feasibility.

The initial population is generated randomly through using a Bernoulli process [37]. For each gene a Bernoulli process is simulated and then the content of the gene is determined.

\subsection{Reproduction, Crossover, and Mutation}

A simple genetic algorithm that yields good results in many practical problems is composed of three operators, namely, reproduction, crossover, and mutation [36]. Reproduction is a process in which individual chromosomes are copied according to their fitness function values. The easiest and the most popular method of reproduction is the one referred to as "roulette wheel method", where each current chromosome in the population has a roulette wheel slot, sized in proportion to its fitness. 


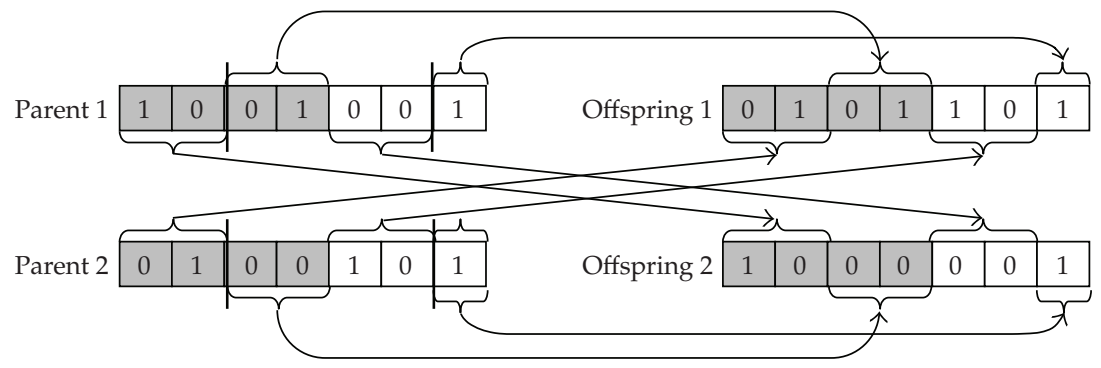

Figure 3: Illustration of the crossover operator.

The crossover operator takes two chromosomes and swaps a part of their genetic information to produce new chromosomes. The easiest and the most classical method for crossover is to choose a random cut-point and generate the offspring by combining the segment of one parent to the left of the cut-point with the segment of the other parent to the right of the cut-point. At the crossover stage, the one-cut crossover is employed twice, one for the parental parts between genes 1 through $J$ and the next time for the parental parts between genes $J+1$ through $J+K$. Figure 3 illustrates how the crossover operator works.

Mutation produces spontaneous random changes in various chromosomes. This genetic operation serves the crucial role of replacing the genes lost from population during the selection process so that they can be tried in a new context, or providing the genes that were not present in the initial population. In the implementation the simplest method is used, in which some genes are selected by a small probability and their content is changed. Adopting the elitist strategy, two best chromosomes are excluded from the crossover procedure and they are directly copied to the next generation. Moreover, the best chromosome is excluded from the mutation procedure. The detailed procedure to generate the next generation is as follows.

Step 1. Copy two best chromosomes to the next generation.

Step 2. Select two chromosomes by the roulette wheel method. If a random number from $[0,1]$ is less than a given crossover probability $P_{c}$, generate two chromosomes by the crossover operator, or copy the two chromosomes directly, otherwise. Repeat this step until the next generation is constructed.

Step 3. For every gene in the chromosomes generated at Step 2 excluding the best chromosome, if a random number from $[0,1]$ is less than a given mutation probability $P_{m}$, then change the content of the gene with the opposite value.

\section{Computational Experiments}

In order to measure the effectiveness of the proposed mathematical model and the adapted GA methodology for real word applications, a number of test problem instances are generated. Table 2 introduces the value of parameters used as constants in the body of the proposed mathematical model. Four problems with increasing size were considered. The values of $A_{i}$ and $D_{i}$ have been selected as randomly distributed in the intervals $[200,500]$ and $[100,200]$, respectively. The size complexity of the problems was depended on the values of $I$ and $J$. The number of demand points (I) was considered at four levels, that is, 20, 40, 60, 
Table 2: Constant parameters and their values.

\begin{tabular}{lccc}
\hline Parameter & Value & Parameter & Value \\
\hline$H$ & 50000 & $\theta_{11}$ & 0.018 \\
$R$ & 100000 & $\theta_{12}$ & 0 \\
$f_{1}$ & 100 & $\theta_{13}$ & 0.003 \\
$f_{2}$ & 50 & $\theta_{21}$ & 0.018 \\
$C_{1}$ & 11 & $\theta_{22}$ & 0.025 \\
$C_{2}$ & 10 & $\theta_{23}$ & 0.02 \\
$\beta_{1}, \beta_{2}$ & 0.5 & $\theta_{15}$ & 0.386 \\
$g$ & 300 & $\theta_{25}$ & 0.937 \\
\hline
\end{tabular}

Table 3: Computational results.

\begin{tabular}{lccccccc}
\hline \multicolumn{7}{c}{ LINGO 9.0 } & \multicolumn{7}{c}{ GA } \\
\hline Problems & $\begin{array}{c}\text { Optimal } \\
\text { objective }\end{array}$ & $\begin{array}{c}\text { Time } \\
(\mathrm{sec})\end{array}$ & Best & Worst & Average & $\begin{array}{c}\text { Time } \\
(\mathrm{sec})\end{array}$ & $\begin{array}{r}\text { Gap } \\
(\%)\end{array}$ \\
\hline Problem 1 & 6545186 & 12 & 6630500 & 7178300 & 6988110 & 39.7 & 6.8 \\
Problem 2 & 15574450 & 2400 & 16398000 & 16812000 & 16591100 & 147.9 & 6.5 \\
Problem 3 & 26775590 & 8880 & 27416000 & 28774000 & 28232000 & 452.7 & 5.4 \\
Problem 4 & 26618180 & 82800 & 27635000 & 30675000 & 28803800 & 1263.3 & 8.2 \\
\hline
\end{tabular}

and 80 . The number of the distribution-collection centers and dismantlers ranged from 10 to 40 at 10 intervals. The values of $L$ and $S$ were equal to 2 and 3 in all investigated problems. Facilities X-coordinates are randomly distributed in the interval $[0,1000]$ and Y-coordinates in $[0,500]$.

The proposed GA methodology was implemented through a computer program written in MATLAB 7.04 and executed on a laptop computer with $2 \mathrm{GHz}$ of CPU speed and 2048 MB RAM. The mathematical model is also implemented in LINGO 9.0 and executed on the same computer. The preliminary computations showed that the choice of $0.5,0.05,10$, and 100 would be suitable for $P_{c}, P_{m}$, the population size, and the number of generations, respectively.

The GA approach was executed 10 times on each of the four problems and the best, worst, and average results are reported here. For each problem, the time taken to conclude the search is also reported. Results are summarized in Table 3. In this table, the columns under the caption of "Lingo 9.0" reflect the optimal value of the objective for the problem and the time taken to verify optimality of the solution found by Lingo 9.0. The column under the title of "Gap" measures the average percentage of distance to the optimal solution.

From Table 3, one can conclude that both GA and Lingo 9.0 could terminate successfully. However, the time taken to solve the problem optimally by Lingo 9.0 is far more than that of required by GA. But for GA, this is at the expense of quality of the obtained solution. So there would be a tradeoff between assuring optimality of the solution by LINGO system and the time saved by GA methodology. But the point is that for large-scale problems there is little hope to solve the problem optimally, and relying upon approximation algorithms like GA would be a reasonable strategy. 


\section{Summary and Conclusion}

The management of the ELVs recovery process and setting up the required infrastructure is receiving increasing attention in developing countries. In this paper, a conceptual model including the production of new vehicles and recovery of used ones is designed. New entities called distribution-collection centers are assumed which are responsible for distributing new vehicles and also collecting the ELVs. The supervision of the whole network is conducted by the vehicle manufacturer. A mathematical model for minimizing the costs of setting up such network and the flow of materials between different facilities is then developed.

The proposed model can help manufacturers to efficiently fulfill the environmental legislations requirements. The model will also enable them to better manage the new vehicles and ELVs transportation routes by sharing the resources. By creating this network, valuable information such as recovery methods and Design For Disassembly advices would be exchanged between different network entities. In an upper tier, the government or the environmental organizations may be involved in designing the recovery network and its different aspects, for example, the monetary transactions.

The high complexity of the model does not permit for developing polynomial time exact algorithms; so a search methodology based on the genetic algorithm is adapted. After selecting a suitable representation of a solution and determining the genetic algorithm operators, a number of test problems were randomly generated and solved. The quality of the generated solutions and the algorithm run time were compared with the results of the LINGO 9.0 software. For large-scale problems in which there is little hope to solve the problem optimally, using the proposed genetic algorithm is a suitable method for finding good quality solutions in reasonable run time.

A number of extensions may be possible to further tightening the gaps reported by the GA. Devising suitable chromosomal representations, which handle concurrent location and allocation decisions, would be an interesting direction. Introducing a low -cost local neighborhood search for improvement of the candidate solutions is also another way of enhancing the GA methodology. Finally, employing other search methods such as tabu search, simulated annealing algorithm, and doing a comparison study would be suitable methods.

\section{Appendix}

\section{Indices}

$i$ : Demand points; $i=1,2, \ldots, I$

$j$ : Distribution-collection centers; $j=1,2, \ldots, J$

$k$ : Dismantling sites; $k=1,2, \ldots, K$

l: Shredders; $l=1,2, \ldots, L$

$s:$ Recyclers; $s=1,2,3$

$m$ : Vehicle type; $m=1,2$ for new vehicle and ELV, respectively

$n$ : ELV type; $n=1,2$ for the ELVs with resale parts and ELVs without resale parts, respectively.

\section{Parameters}

$H$ : The fixed cost of opening a distribution-collection center 
$f_{m}$ : The variable cost (per weight unit) of opening a distribution-collection center related to vehicle type $m$

$R$ : The fixed cost of opening a dismantling site

$g$ : The variable cost (per weight unit) of opening a dismantling site

$d_{1 i j}$ : Distance between $i$ th demand point and $j$ th distribution-collection center

$d_{2 i k}$ : Distance between $i$ th demand point and $k$ th dismantling site

$d_{3 j k}$ : Distance between $j$ th distribution-collection center and $k$ th dismantling site

$d_{4 k l}$ : Distance between $k$ th dismantling site and $l$ th shredder

$d_{5 k s}$ : Distance between $k$ th dismantling site and recycler type $s$; [ 1 for fluids, 2 for tyres and 3 for plastics recycler]

$d_{j}$ : Distance between manufacturer and $j$ th distribution-collection center

$C_{m}$ : Transportation cost of a weight unit of vehicle type $m$ per distance unit

$A_{i}$ : Amount of ELVs in demand point $i$ in weight unit

$D_{i}$ : Amount of demand for new vehicle in demand point $i$ in weight unit

$C_{j m}$ : Maximum potential capacity of distribution-collection center $j$ related to vehicle type $m$ in weight unit

$E_{k}$ : Maximum potential capacity of dismantling site $k$ in weight unit

$B_{j}$ : The available budget for setting up distribution-collection center $j$

$\beta_{n}$ : Percentage of ELVs type $n$ in dismantling sites

$\theta_{n 1}$ : Percentage of fluids from the vehicle weight, for ELVs type $n$

$\theta_{n 2}$ : Percentage of tyres from the vehicle weight, for ELVs type $n$

$\theta_{n 3}$ : Percentage of removed non-resalable plastic parts from the vehicle weight, for ELVs type $n$

$\theta_{n 4}$ : Percentage of disassembled parts for reuse or remanufacture from the vehicle weight, for ELVs type $n$; including resalable plastic parts

$\theta_{n 5}$ : Percentage of the ELVs type $n$, which is sent to the shredder (hulk).

\section{Decision Variables}

$X_{j}$ : A binary variable which is equal to 1 if distribution-collection center $j$ is selected for opening and 0 otherwise

$Y_{k}$ : A binary variable which is equal to 1 if dismantling site $k$ is selected for opening; and 0 otherwise

$t_{1 i j}$ : Amount of ELVs transported from $i$ th demand point to $j$ th distribution-collection center in weight unit

$t_{2 i k}$ : Amount of ELVs transported from $i$ th demand point to $k$ th dismantling site in weight unit

$t_{3 j k}$ : Amount of ELVs transported from $j$ th distribution-collection center to $k$ th dismantling site in weight unit

$t_{4 k l}$ : Amount of ELVs transported from $k$ th dismantling site to $l$ th shredder in weight unit 
$t_{j}$ : Amount of new vehicles transported from manufacturer to $j$ th distributioncollection center in weight unit

$u_{j}$ : Capacity of distribution-collection center $j$ related to ELVs in weight unit (dependent variables)

$v_{k}$ : Capacity of dismantling site $k$ in weight unit (dependent variables).

\section{References}

[1] A. Gungor and S. M. Gupta, "Issues in environmentally conscious manufacturing and product recovery: a survey," Computers $\mathcal{E}$ Industrial Engineering, vol. 36, no. 4, pp. 811-853, 1999.

[2] European Council, "Directive 2000/53/EC of The European Parliament and of the Council of 18 September 2000 on end-of life vehicles," Official Journal of the European Communities, vol. 269, pp. 3442, 2000.

[3] D. S. Rogers and R. S. Tibben-Lembke, Going Backwards: Reverse Logistics Trends and Practices, Reverse Logistics Executive Council, University of Nevada, Reno, Nev, USA, 1998.

[4] C. Edwards, G. Coates, S. Rahimifard, T. Bhamra, S. Newman, and P. Leaney, "End-of-life recovery of vehicles in the UK," in Proceedings of the 4th International Conference on Design and Manufacture for Sustainable Development (Sustain05), Newcastle, UK, 2005, paper \# Man1-3.

[5] R. Cruz-Rivera and J. Ertel, "Reverse logistics network design for the collection of end-of-life vehicles in Mexico," European Journal of Operational Research, vol. 196, no. 3, pp. 930-939, 2009.

[6] D.-H. Lee and M. Dong, "A heuristic approach to logistics network design for end-of-lease computer products recovery," Transportation Research Part E, vol. 44, no. 3, pp. 455-474, 2008.

[7] H. R. Krikke, A. Van Harten, and P. C. Schuur, "On a medium term product recovery and disposal strategy for durable assembly products," International Journal of Production Research, vol. 36, no. 1, pp. 111-139, 1998.

[8] P. Ferrão and J. Amaral, "Assessing the economics of auto recycling activities in relation to European Union Directive on end of life vehicles," Technological Forecasting and Social Change, vol. 73, no. 3, pp. 277-289, 2006.

[9] A. P. Bandivadekar, V. Kumar, K. L. Gunter, and J. W. Sutherland, "A model for material flows and economic exchanges within the U.S. automotive life cycle chain," Journal of Manufacturing Systems, vol. 23, no. 1, pp. 22-29, 2004.

[10] M. R. Johnson and M. H. Wang, "Evaluation policies and automotive recovery options according to the European Union Directive on end-of-life vehicles (ELV)," Proceedings of the Institution of Mechanical Engineers, Part D, vol. 216, no. 9, pp. 723-739, 2002.

[11] J. Gerrard and M. Kandlikar, "Is European end-of-life vehicle legislation living up to expectations? Assessing the impact of the ELV Directive on 'green' innovation and vehicle recovery," Journal of Cleaner Production, vol. 15, no. 1, pp. 17-27, 2007.

[12] Z. Lu and N. Bostel, "A facility location model for logistics systems including reverse flows: the case of remanufacturing activities," Computers E Operations Research, vol. 34, no. 2, pp. 229-323, 2007.

[13] A. I. Barros, R. Dekker, and V. Scholten, "A two-level network for recycling sand: a case study," European Journal of Operational Research, vol. 110, no. 2, pp. 199-214, 1998.

[14] V. Jayaraman, R. A. Patterson, and E. Rolland, "The design of reverse distribution networks: models and solution procedures," European Journal of Operational Research, vol. 150, no. 1, pp. 128-149, 2003.

[15] S. K. Srivastava, “Network design for reverse logistics," Omega, vol. 36, no. 4, pp. 535-548, 2008.

[16] A. Alshamrani, K. Mathur, and R. H. Ballou, "Reverse logistics: simultaneous design of delivery routes and returns strategies," Computers E Operations Research, vol. 34, no. 2, pp. 595-619, 2007.

[17] J.-B. Sheu, "A coordinated reverse logistics system for regional management of multi-source hazardous wastes," Computers \& Operations Research, vol. 34, no. 5, pp. 1442-1462, 2007.

[18] T.-L. Hu, J.-B. Sheu, and K.-H. Huang, "A reverse logistics cost minimization model for the treatment of hazardous wastes," Transportation Research Part E, vol. 38, no. 6, pp. 457-473, 2002.

[19] D. Louwers, B. J. Kip, E. Peters, F. Souren, and S. D. P. Flapper, "A facility location allocation model for reusing carpet materials," Computers $\mathcal{E}$ Industrial Engineering, vol. 36, no. 4, pp. 855-869, 1999. 
[20] A. Marín and B. Pelegrín, "The return plant location problem: modelling and resolution," European Journal of Operational Research, vol. 104, no. 2, pp. 375-392, 1998.

[21] F. Schultmann, M. Zumkeller, and O. Rentz, "Modeling reverse logistic tasks within closed-loop supply chains: an example from the automotive industry," European Journal of Operational Research, vol. 171, no. 3, pp. 1033-1050, 2006.

[22] A. Nagurney and F. Toyasaki, "Reverse supply chain management and electronic waste recycling: a multitiered network equilibrium framework for e-cycling," Transportation Research Part E, vol. 41, no. 1, pp. 1-28, 2005.

[23] G. Fandel and M. Stammen, "A general model for extended strategic supply chain management with emphasis on product life cycles including development and recycling," International Journal of Production Economics, vol. 89, no. 3, pp. 293-308, 2004.

[24] O. Listeş and R. Dekker, "A stochastic approach to a case study for product recovery network design," European Journal of Operational Research, vol. 160, no. 1, pp. 268-287, 2005.

[25] P. Georgiadis and D. Vlachos, "The effect of environmental parameters on product recovery," European Journal of Operational Research, vol. 157, no. 2, pp. 449-464, 2004.

[26] M. Fleischmann, H. R. Krikke, R. Dekker, and S. D. P. Flapper, "A characterisation of logistics networks for product recovery," Omega, vol. 28, no. 6, pp. 653-666, 2000.

[27] M. P. de Brito and R. Dekker, "A framework for reverse logistics," Report Series Research in Management, Erasmus Research Institute of Management, 2003.

[28] J.-E. Lee, M. Gen, and K.-G. Rhee, "Network model and optimization of reverse logistics by hybrid genetic algorithm," Computers E Industrial Engineering, vol. 56, no. 3, pp. 951-964, 2009.

[29] H. Min, H. J. Ko, and C. S. Ko, "A genetic algorithm approach to developing the multi-echelon reverse logistics network for product returns," Omega, vol. 34, no. 1, pp. 56-69, 2006.

[30] H. Min, C. S. Ko, and H. J. Ko, "The spatial and temporal consolidation of returned products in a closed-loop supply chain network," Computers $\mathcal{E}$ Industrial Engineering, vol. 51, no. 2, pp. 309-320, 2006.

[31] H. J. Ko and G. W. Evans, "A genetic algorithm-based heuristic for the dynamic integrated forward/reverse logistics network for 3PLs," Computers \& Operations Research, vol. 34, no. 2, pp. 346 366, 2007.

[32] C. K. M. Lee and T. M. Chan, “Development of RFID-based Reverse Logistics System," Expert Systems with Applications, vol. 36, no. 5, pp. 9299-9307, 2009.

[33] K. Lieckens and N. Vandaele, "Reverse logistics network design with stochastic lead times," Computers $\mathcal{E}$ Operations Research, vol. 34, no. 2, pp. 395-416, 2007.

[34] J. Bautista and J. Pereira, "Modeling the problem of locating collection areas for urban waste management. An application to the metropolitan area of Barcelona," Omega, vol. 34, no. 6, pp. 617629, 2006.

[35] S. Mansour and M. Zarei, "A multi-period reverse logistics optimisation model for end-of-life vehicles recovery based on EU Directive," International Journal of Computer Integrated Manufacturing, vol. 21, no. 7, pp. 764-777, 2008.

[36] M. Gen and R. Cheng, Genetic Algorithms and Engineering Design, Wiley, New York, NY, USA, 1997.

[37] C. W. Helstrom, Probability and Stochastic Processes for Engineers, Macmillan, New York, NY, USA, 1984. 


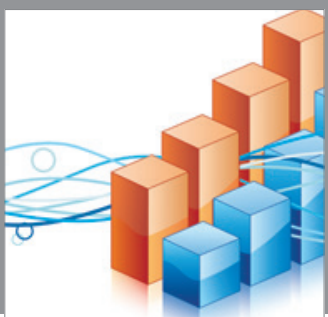

Advances in

Operations Research

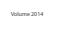

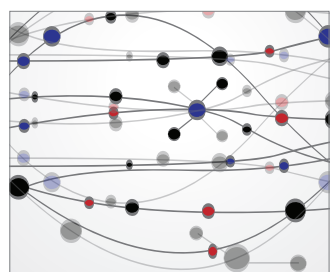

\section{The Scientific} World Journal
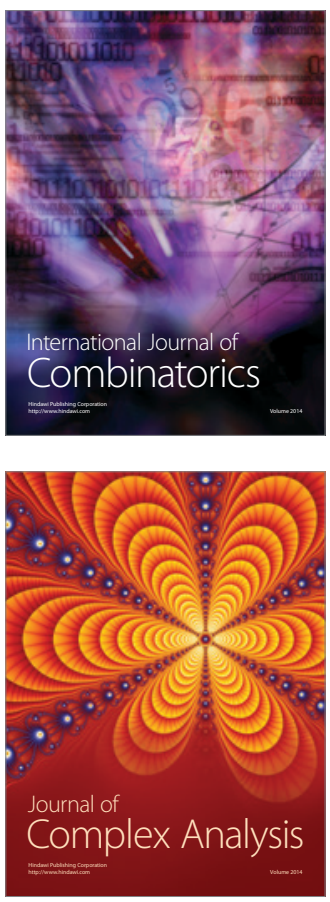

International Journal of

Mathematics and

Mathematical

Sciences
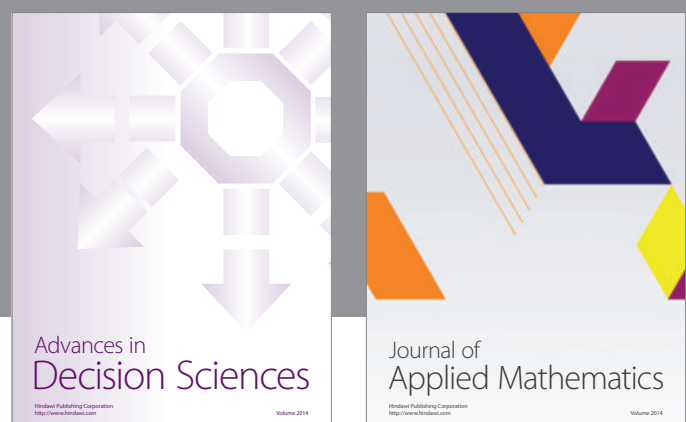

Journal of

Applied Mathematics
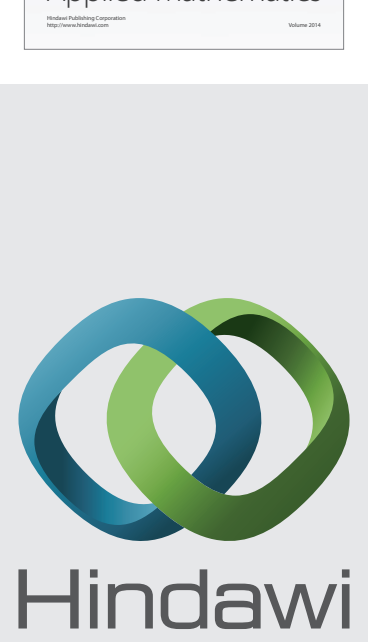

Submit your manuscripts at http://www.hindawi.com
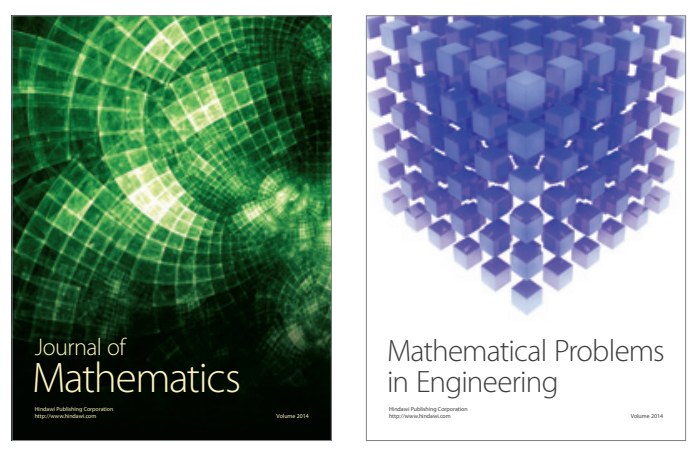

Mathematical Problems in Engineering
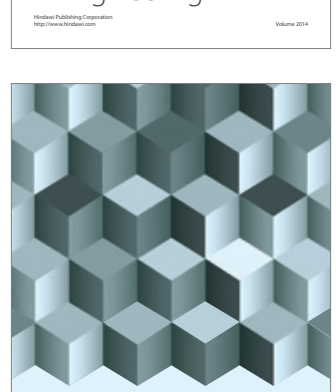

Journal of

Function Spaces
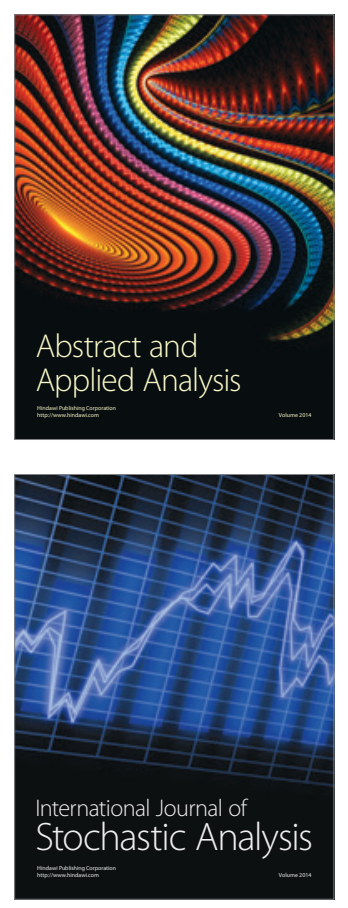

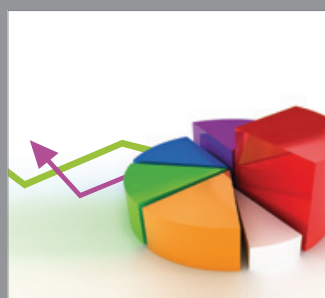

ournal of

Probability and Statistics

Promensencen
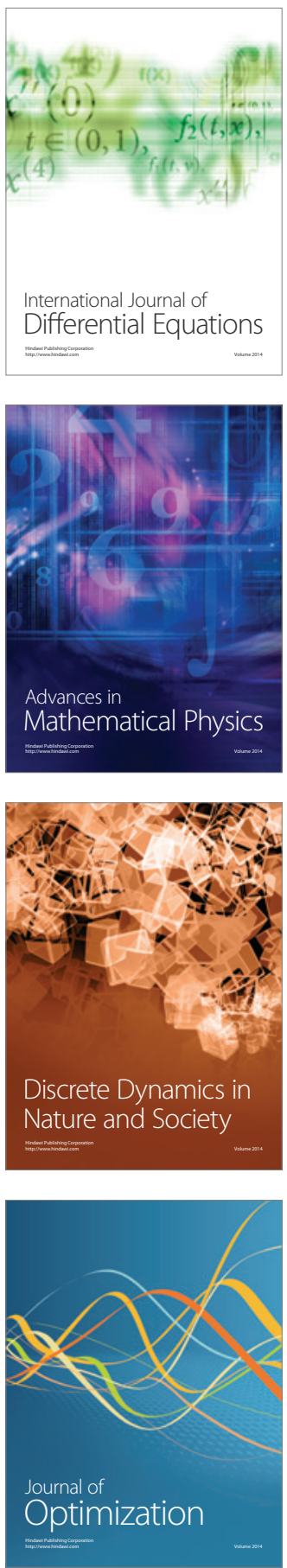